\title{
Adoption and Appropriation: A Design Process from HCI Research at a Brazilian Neurological Hospital
}

\author{
Junia Anacleto ${ }^{1}$ and Sidney Fels ${ }^{2}$ \\ ${ }^{1}$ Department of Computer Science, Federal University of Sao Carlos, Sao Carlos, Brazil \\ ${ }^{2}$ Department of Electrical and Computer Engineering, University of British Columbia, \\ Vancouver, BC, Canada \\ junia@dc.ufscar.br, ssfels@ece.ubc.ca
}

\begin{abstract}
Through our research on natural ICT solutions for integration into a non-ICT based workflow at a Brazilian chronic care hospital, we created a new design process and two additional $\mathrm{HCI}$ design criteria for maintaining natural work processes using information and communication technologies (ICT). For our HCI design we propose two design pathways: 1. iterating on adoption of designed technologies and 2. iterating on appropriation of these technologies. The degree of appropriation provides an indicator of how natural a design is since it allows for users' inventiveness to uncover latent affordances for use in new contexts. Thus, the use of an interface along with whether its potential is realized in new, user-oriented contexts, are critical elements for designing natural interfaces. We report our insights gained through observations and usercentered design for health professionals at a non-ICT based, large chronic-care hospital to support this perspective.
\end{abstract}

Keywords: human computer interaction, appropriation, adoption, design process, user centered design, health care, mental illness, non-disruptive workflow.

\section{Introduction}

We have partnered with the Brazilian Hospital CAIS Clemente Ferreira, that is a special chronic care hospital for individuals with neurological and brain disorders. A primary objective of the Hospital is for professional health staff to facilitate patients' transitioning from the hospital back into normal society. The hospital has 3 floors with 6 wings with 800 patients and 600 professionals distributed throughout. The hospital has four distinct professional roles: administration, health professionals, nurse assistants and maintenance. Currently the Hospital has essentially no Wi-Fi or cell phone coverage due to architectural issues (at least $70 \mathrm{~cm}$ thick concrete walls) and budget (no funds to install Wi-Fi service). They use voice and a paper-based system for their primary workflow mechanisms. Our primary research project is targeted towards the health professionals and not the patients. We believe that working with these professionals is an opportunity to design information technology based workflow that avoids a WIMP oriented strategy. Likewise, we can use their 
workflow as a starting point in deciding what natural is, given that their workflow has evolved over more than two decades of practice. Thus, we argue that introducing unnatural technologies into this workflow should be obvious if we see a substantial disruption.

We began our design for the hospital looking to gesture and speech based technologies as they promise to enable computers to understand people's naturally evolved and learned methods of interactions. Further, in some environments where the existing Windows, Icons, Menus and Pointers (WIMP) approach doesn't match many of the workflow activities, alternative approaches that look towards gesture and speech interaction may make more sense. In particular, in our hospital environment, most of the health care workers are nomadic. Thus, designing to free the professionals from workstation/WIMP based solutions appears to be a logical fit. However, during our user-centred design process to construct these WIMP alternative solutions, we identified two main challenges: 1. There is not a design process to follow for maintaining natural workflow and 2. It is unclear what natural is and how to measure it. This paper addresses both of these issues through our research on user interface design with health care professionals in a hospital in Brazil. We look for natural ICT solutions, independently of the adopted interaction paradigm. We begin with related work followed by our modified user-centred design process model in relation to some well-established WIMP based approaches. Next, we describe the research, leading to a discussion and analysis supporting our proposed design process. We provide conclusions and future work at the end.

\section{Related Work}

Recently, many studies, such as $[2,3,11]$, have been carried out for finding ICT, and WIMP based solutions especially, within hospital settings with many different objectives, like: improving communication processes among hospital staff, providing more accurate diagnostic tools and treatments, assist in therapy processes, increase patient's medication adherence, and others. These efforts are categorized in the eHealth concept encompassing various initiatives to use these technologies to improve health processes. In general, e-Health has been defined as the use of internet and other technologies in the health sector to improve access, efficiency, effectiveness and quality of clinical processes used by health organizations, patients and consumers in an attempt to improve the health of patients [12]. Thus, while we are considering ICT solutions for health care workers, we also seek to enable hospital staff to evolve their practices to determine how best to adopt some of the success that may apply to them.

\section{$3 \quad$ Natural ICT Design Process}

Our proposed design process, shown in figure 1, includes both adoption and appropriation activities. We consider adoption as the process of users learning and accepting a designed solution for some of their activities. In contrast, we look as appropriation as the use of an artifact designed for one type problem, to solve a 
separate one. We arrived at this process by looking to define natural interface design to have two properties: 1. the introduction of technology should not interrupt existing workflow and 2. that the adopted technology must also be shown to be appropriated for use in other contexts. To accommodate these properties, in our HCI design process, we added an additional phase to the usual design-prototype-evaluate design process [10]. From our observations designing for the Hospital, we determined that it is necessary to support users to first pass through the adoption phase so that they perceiving the affordances of a developed solution or prototype. Then, include affordances and mechanisms for supporting the appropriation phase where they can envision the use of such a solution in a new context.

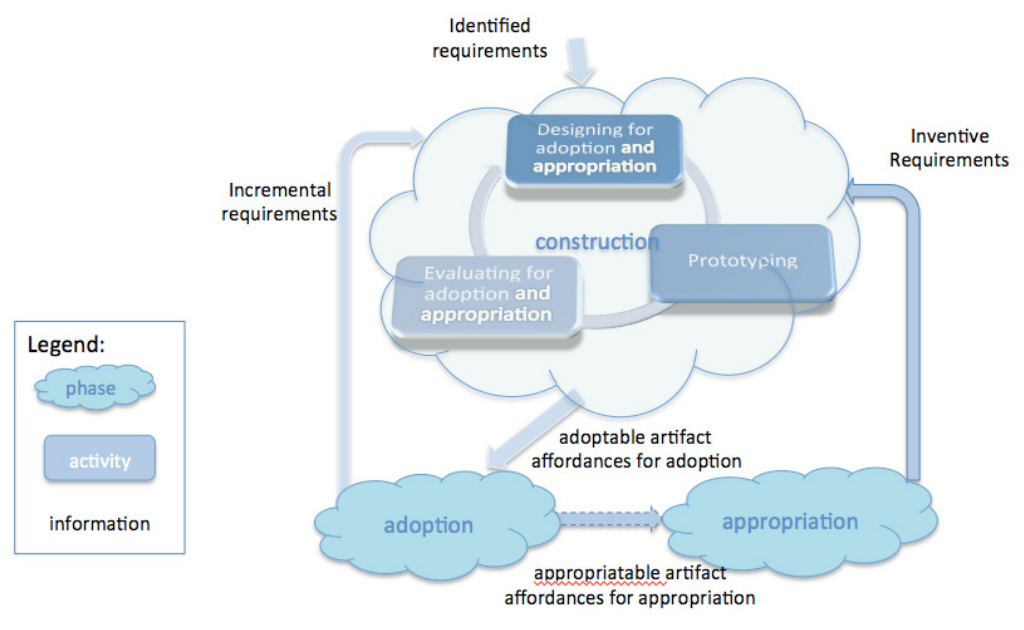

Fig. 1. Natural ICT Solution Design Process

Our development process starts with a Construction phase triggered by some initial requirements. We envision this phase including established HCI user-centered development (UCD) processes such as covered in [10]. Traditionally, the criteria for successful design in the construction phase for WIMP interfaces focuses on usability and usefulness to improve the chances that an artifact will be adopted by the target user community for a specified set of tasks. For a hospital context, researchers $[5,6,7,9]$, have used a 3-phase design-prototype-evaluate approach. For example, we employed participatory design, ethnography, work place shadowing and other UCD design strategies during our work. Each iteration within the Construction phase leads to a release of an ICT artifact. Once the artifact is created, the next step is deployment and adoption by the target user group. This could be either a small test group or the expected target population. Once adopted, new requirements arise from the users to improve the system to solve the problem that the system was designed for. These normally come as incremental requirements to adjust the interaction design to allow for a gradual refinement to a well-designed system to solve user's problems that they 
can adopt easily. The systematic adoption evaluation activity in the construction phase attempts to discover these requirements to increase the effectiveness of adoption. In UCD, this process continues from the early stages of construction to the complete lifecycle of the product. Our observations suggest that while the process, up to this point, leads to establishing new methods to iteratively improve adoption of solutions to a particular problem, it does not include any method to facilitate appropriation. As described in section 4, in our research, this appropriation is critical as it is part of how the Hospital professionals have evolved their work practices. Thus, we add the appropriation phase as part of the development cycle for maintaining natural workflows. During the adoption phase, a user (or possibly users) becomes comfortable enough with the technology that he or she may appropriate the design for a new context to solve problems unforeseen during the Construction phase.

Once appropriation occurs, the user creates new requirements based on the new context of use and the appropriated solution. These are inventive requirements. The appropriation evaluation in the Construction phase can then establish these new, inventive requirements to feed into a new design. The design may be an entirely new product tailored to solve the problems in the new context in a new way. We identify some of the appropriation design approaches that facilitate this process in section 5 . Alternatively, the designers may adjust the next version of the product to expand the contexts that it may be used in.

In summary, the Natural ICT Design process leads to designs that both support adoption and appropriation. For our work, we are applying our approach to gesturebased, mobile solutions with the additional constraint that it should impose minimal disruption in the existing workflow to be natural. However, we suggest that the Natural ICT Design approach is also appropriate for ICT solutions where the aim is to support the natural evolution of work practices. As we discuss in the next section, our approach progress as we designing solutions in the Hospital.

\section{Our Design Process in the Hospital}

We have used been using UCD design process as described in the previous section that currently has been ongoing from June 15, 2011 to now. During this time, we have had 9 half-day or full day meetings with healthcare staff, documented and validated their workflow, established 4 scenarios, and created 3 prototypes for testing, including a long term study that is ongoing. As well, we applied a survey and a one week workplace shadowing activity.

Our process began with a survey and initial discussions with the Director of the Hospital to initialize the Construction phase. Within the Construction phase we used both Participatory design (PD) and task-centred design for design methods as well as low and medium prototyping and various evaluation methods. A group of six trained health professionals (Figure 2) with extensive experience were part of the PD team: 1 physiotherapist (15+ years experience, undergraduate degree), 2 nurses (20+ years of experience, nursing degree), 1 occupational therapist $(15+$ years experience, undergraduate degree), 1 social assistant. Two professionals never used e-mail or any 
social network and never bought something via internet; two use iPods and demonstrated abilities with ICT; all professionals had contact with touch technology, mobile phones and web search; none of them had contact with free-form gesture based technology. Our choice of PD was intended to avoid our own bias towards the

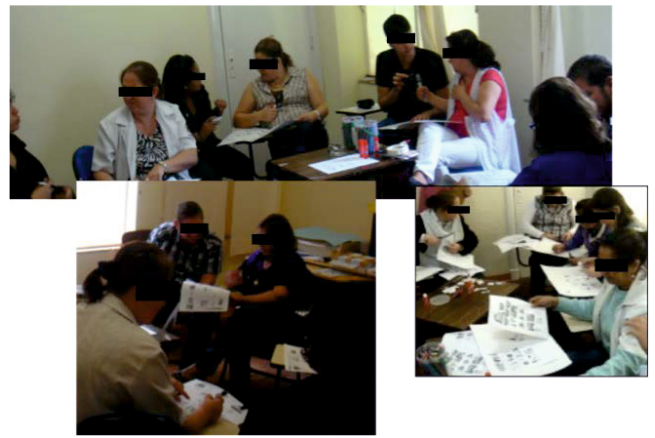

Fig. 2. PD activity with health professionals use of WIMP interaction for some of the scenarios that were identified. We continued with PD-prototypingevaluation until we arrived at five scenarios leading to three mediumfidelity prototypes.

The first prototype involved placing public displays in common workrooms that allowed the Hospital Director to put a message to call emergency meetings. The prototype worked by having the Director's assistant make a phone call to the rooms when a meeting is called (the current practice) to let people know there is a meeting; however, for rooms with our prototype, the person who answered the phone pressed a large red button displayed on the screen (using a mouse pinned on the wall) that displayed a large message that a meeting has been called that would stay on screen for $30 \mathrm{~m}$. We deployed this in one wing and used the current verbal relay method in another wing as a control. The second prototype had two variations due to the changing nature of the physiotherapist's scenario. It used a Kinect ${ }^{\mathrm{TM}}$ to sense body posture and provided limb, head and neck angles for use in the prototype systems. This device was used for prototyping both physiotherapists' scenarios: the game distraction system and the hand's free goniometer capture system. The third prototype was a simple ticketing system that used the public displays. Health care professionals could generate a request for help by typing in a message that would appear on the screen. Then, someone seeing that message, could help out and then flag the request as attended to. We had two different public visualizations of the usage of the system to see whether competitive or cooperative representations would help with enhancing the adhoc helping mechanisms that were identified as key elements of the success of the functioning of the hospital.

One important observation made during the design session with the physiotherapist (Feb 23, 2012) is that she disclosed that she had recently used the camera on her cell phone to take photos of one of her patients recovering from an injury. She was planning to some of the photos at a conference. However, she decided to print the photos and put them in a photo album documenting healing progression. One day, she showed the

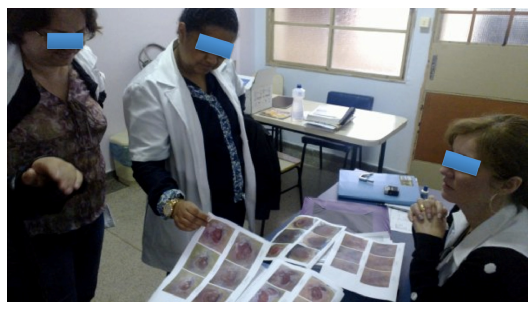

Fig. 3. Photos using personal cellphone to document physiotherapy results album to her colleagues that stimulated 
discussion and how they could use this approach for their patients (Figure 3). As discussed below, this is an example of appropriation of the technology, suggesting that the camera interface itself has natural properties.

\section{$5 \quad$ Analyses and Discussion}

Through the 21 months of design activities, we observed situations that led us to argue for two main points about designing natural ICT solutions: 1. the process must include design for appropriation and 2. it should minimize disruption to the existing workflow. To meet these criteria, we argue that the notion of a natural interface provides both Norman's sense of affordances [4] and Gibson's affordances [1]. As pointed out in [8], Norman affordances provide visual cues as to the function of a particular interface that match users common sense knowledge of use. This helps the process of adoption and also leads to less learning required. In contrast, Gibson affordances are the latent functions that an object can support. The typical example provided is that a chair has clear visual affordances that it can be used to sit on; a Norman affordance. However, there are other functions, such as standing on, that may not be visually obvious, but that the chair can support. These would be Gibson affordances as the chair affords making the user taller. Typical, WIMP interfaces focus on Norman affordances for their functions but tend not to afford other functions making it difficult for users to appropriate to new contexts.

In our design process, including Norman affordances supports the design for adoption and leads to easier to use interfaces. Though, if designing ICT solutions, the designer still needs to be concerned with how much disruption the artifact has with the current workflow. For the design for appropriation, however, Gibson affordances are important. As a user encounters new contexts in their activities, there is a natural inclination to appropriate objects designed for one purpose for use in the new one. The ability to do this depends upon the object having the potential to be used in the new context and the user to perceive this. By designing Gibson affordances into the artifact so that objects have the potential to be used for different purposes, this natural evolution is supported. Or conversely, part of the measure of naturalness, is whether the designed objects are appropriated for use in contexts other than what the designed Norman affordances are for. This insight leads to the two design pathways for natural ICT design, as shown in Figure 1.

Designing for appropriation remains a challenge. Dix's basic studies on appropriation [13], suggests that designers should accept that we do not understand completely what will happen in real use with ICT solutions. He argues this is especially true within the context of non ICT-based workflow. Nonetheless, based on our Natural Design process, it is necessary that the ICT solutions are designed so that they can be used in unexpected ways. Further, they need to address the natural environment dynamics so that designing for use is designing for change. To accommodate this, we suggest, the construction phase of design should embody the principles for appropriation listed in Table 1. We refine Dix's principles for natural ICT solutions as that is the direction we are pursuing at the Hospital, however we look to future work to determine if they generalize. Our studies and preliminary 
observations at the Hospital reinforce that appropriation is related to users being comfortable enough with technology to use it in their own ways. We encountered: one successful, one partially successful and one failed appropriation. Specifically, the one success was when the physiotherapist used her camera phone to begin documenting her patient's healing process, then printing the timeline and bringing it to share with her colleagues at the Hospital. This use led to others in the same wing trying the same idea (Figure 3). The camera interface on her cell phone was simple: start the camera application, point and shoot. However, the printing and organizing required significant time and effort to do. The physiotherapist had mastered use of this camera for her personal activities. What we observed is that she made the connection between the capabilities of accumulating a chronology of photos to watch the healing process and then made the leap to see this would be useful for the care of this patient. Our second prototype used a Kinect ${ }^{\mathrm{TM}}$ during physiotherapy as we determined that the physiotherapist identified a problem when she takes care of two children at the same time. After 3 iterations of design, a Kinect-based application was designed, approved by the therapist, and delivered. However, once she tried the actual first functional prototype, with the all the limitations of the Kinect ${ }^{\mathrm{TM}}$, she lost interest in the approach and changed her mind about the whole scenario. We believe, after discussion with her, continuing another scenario, and analyzing video, that there is a substantial gap between what she thought the technology would do and what it would actually do. We concluded that this design supports adoption but it did not lead to appropriation, as she was able to use it but did not see anything beyond the limits of the technology. Our design for a meeting calling scenario was neither successful for adoption nor appropriation. The deployed functional prototype was successfully executed with messages appearing on a public display, but the public displays were completely ignored. Hence, the construction cycle lead to technologies supported by the users during PD but ultimately, were rejected.

Table 1. Principles for Designing for Appropriation (derived from [13])

\begin{tabular}{|c|c|}
\hline Principle & Description \\
\hline Allowing interpretation & Including elements where users can add their own meanings \\
\hline Providing visibility & $\begin{array}{l}\text { It is often the irrelevant state and internal process that can be } \\
\text { appropriated. }\end{array}$ \\
\hline Exposing intentions & $\begin{array}{l}\text { Deliberately exposing the intention behind the system. } \\
\text { Appropriations may subvert the rules of the system }\end{array}$ \\
\hline Supporting, not controlling & $\begin{array}{l}\text { Designing a system so that the task can be done instead of a } \\
\text { system to do the task. } \\
\text { Providing the necessary functions so that the user can achieve } \\
\text { the task (instead of driving the user through the steps). }\end{array}$ \\
\hline $\begin{array}{l}\text { Respecting plugability } \\
\text { and configuration }\end{array}$ & $\begin{array}{l}\text { Designing systems where the user can plug the parts together } \\
\text { in different ways. }\end{array}$ \\
\hline Encouraging sharing & Designing systems that support sharing appropriations \\
\hline $\begin{array}{l}\text { Learning from } \\
\text { appropriation }\end{array}$ & $\begin{array}{l}\text { Observing that a temporary use of a tool has become } \\
\text { specialized. } \\
\text { Observing that a crystalized appropriation leads to a new tool. }\end{array}$ \\
\hline
\end{tabular}




\section{Conclusions}

Through our partnership with the Hospital we have access to a large number of highly trained health professionals who mainly use a verbal and paper based system for managing and care-taking of chronic mental patients. They have evolved their work practices over more than two decades providing an exceptionally rich environment for us to study how they have created natural workflows without technology. As we progressed through our design processes for improving their work practices, we reoriented our thinking to realize that to be able to design ICT solutions for them, we first needed to design technologies that had no impact on their natural work practice. Then, we could begin to work with them to establish how to appropriate the technologies into improving their practices. However, without their inventiveness in the process, the solutions ended up alienating them from being able to improve their care giving potential. Further, we suspect that without this process, trying to introduce technologies from the existing research to allow them to use accepted best practices will generally fail. Thus, we developed an additional element in the design process that encapsulated the natural ability of the health professional to use objects around them, once they had adopted them. We believe this is a critical component in developing natural user interface solutions for health care workers in this context. We look to future work to see how effective it is and whether it generalizes to other contexts.

Acknowledgements. We thank FAPESP, CAPES-DFAIT, Boeing. CAIS Clemente Ferreira Ethics.

\section{References}

1. Gibson, J.J.: The Ecological Approach to Visual Perception, NJ, USA, L. E. Ass (1979)

2. Vuong, C., Ingalls, T., Abbas, J. Transforming clinical rehabilitation into interactive multimedia. In: ACM MM, pp. 1-2, (2011).

3. Geurts, L., et al.: Digital games for physical therapy: fulfilling the need for calibration and adaptation. In: TEI 2011, pp. 117-124 (2011)

4. Norman, D.: Affordances, Conventions, and Design. Interactions 6(3), 38-41 (1999)

5. Bath, P.A.: Health informatics: current issues and challenges. J. Inf. Sci. 34-4, 501-518 (2008)

6. Li, J., Wilson, L., Stapleton, S., Cregan, P.: Design of an advanced telemedicine system for emergency care. In: OZCHI 2006, pp. 413-416 (2006)

7. Reeder, B., Turner, A.: Scenario-based design: A method for connecting information system design with public health ops and emerg mgmt. J. Bio. Inform. 44(6), 978-988 (2011)

8. McGrenere, J., Ho, W.: Affordances: Clarifying and Evolving a Concept. In: GI 2000, pp. 179-186 (2000)

9. Norman, D.A.: Natural User Interfaces are Not Natural. Interactions 17(3), 6-10 (2010)

10. Preece, J., Rogers, Y., Sharp, H.: Interaction Design, 1st edn. John Wiley \& Sons, Inc., New York (2002)

11. Schönauer, C., et al.: Chronic pain rehabilitation with a serious game using multimodal input. In: Virtual Rehabilitation (ICVR), pp. 1-8 (2011)

12. Broderick, M., Smaltz, D.: HIMSS E-Health White Paper: e-Health Defined (2008), http: / /www.himss.org/content/files/ehealth_whitepaper.pdf

13. Dix, A.: Designing for appropriation. BCS HCI (2), 27-30 (2007) 